\title{
Single-vision spectacle use and myopia progression in children with low myopia, a propensity score matching study
}

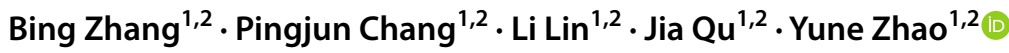

Received: 28 July 2021 / Revised: 10 September 2021 / Accepted: 19 September 2021 / Published online: 9 October 2021

(c) The Author(s), under exclusive licence to Springer-Verlag GmbH Germany, part of Springer Nature 2021

\begin{abstract}
Purpose To investigate the impact of single-vision spectacle use on myopia progression in children with low myopia. Methods MYOSOTIS is a prospective myopia screening survey including all 46 primary and junior high schools in two districts of Hangzhou, China. After 1-to-1 propensity score matching (PSM), 1,685 pairs of students with low myopia were included. Group 1 was composed of 1,685 non-spectacle users at baseline, and group 2 consisted of 1,685 spectacle wearers at both survey rounds. Refraction was examined by noncycloplegic autorefraction and mean spherical equivalent refraction (SER) of both eyes was analyzed. Myopia progression was measured by average rate of change in SER ( $\mathrm{r} \triangle \mathrm{SER}$ ) between two survey rounds and compared between the two groups. Results After PSM, no significant difference in age, sex ratio, SER, and uncorrected visual acuity (VA) between the two groups was found at baseline. For myopic progression, $\mathrm{r} \triangle \mathrm{SER}$ showed no significant difference between the two groups $(-0.67 \pm 0.97$ versus $-0.69 \pm 0.81$ diopter/year, $P=0.448)$. After adjusting for age, sex, SER, and VA, the difference in $\mathrm{r} \triangle \mathrm{SER}$ between the two groups was not significant $(-0.031,95 \% \mathrm{CI}-0.089 \sim 0.028$ diopter/year, $P=0.302)$. In the subgroup analyses stratified by age and SER, and in the sensitivity analyses by eye side, there was still no significant difference in myopia progression between the two groups.

Conclusions Our study indicates that single-vision spectacle use has no impact on myopia progression in children with low myopia. Spectacles are recommended in children with low myopia if their visual acuity has interfered with the daily life.
\end{abstract}

Keywords Single-vision spectacle use $\cdot$ Myopia progression $\cdot$ Children and teenagers $\cdot$ Propensity score matching . Refractive error

\section{Key messages}

- The impact of single-vision spectacle use on myopia progression is a concern to many parents when their children become near-sighted, but its impact on myopia progression in children remains unclear.

- This study indicates single-vision spectacle use has no impact on myopia progression in children with low myopia.

- Spectacle use is recommended in children with low myopia if their visual acuity has interfered with the daily life.

Jia Qu

jia.qu@163.com

$\triangle$ Yune Zhao

zyehzeye@126.com

1 Eye Hospital and School of Ophthalmology and Optometry, Wenzhou Medical University, 270 Xueyuan Road, Wenzhou 325027, Zhejiang, China

2 National Clinical Research Center for Ocular Diseases, Wenzhou, Zhejiang, China

\section{Background}

Myopia is a growing global health burden and it is predicted that nearly half $(49.8 \%)$ of the world population will be near-sighted by 2050 [1]. Uncorrected myopia is a major and amenable cause for visual impairment globally [2]. Single-vison spectacle use is generally considered a simple and safe approach to correct refractive error. 
However, the impact of spectacle use on myopia progression remains unclear, since it is usually studied as negative control in myopia control trials and its impact could be masked as a control group [3].

One widespread and highly controversial opinion is that spectacle use may cause faster myopia progression [3, 4]. This may discourage some children with low myopia from spectacle use, especially when they have never worn glasses before. In history, spectacle use has been suspected to cause higher degree of myopia for over a century, especially in the condition of full correction [4]. However, studies in recent years, which compared the myopia progression between participants with under and full correction, showed no significant difference between the two conditions [5], which indicates that the belief that spectacle use may accelerate myopia progression can also be incorrect.

To the best of our knowledge, there is lack of evidence on the relationship between myopia progression and singlevision spectacle use. One reason is that it is highly impossible to perform an intervention study to keep the myopia children uncorrected, which can possibly decrease their quality of life [6] as well as academic performance [7]. For observational study, the remarkable differences in refractive state, age, and visual acuity between spectacle users and non-users can significantly bias the outcomes and make the conclusion unreliable.

Propensity score matching (PSM) is a method to reduce the bias in estimating treatment effects by assembling similar groups with different treatments [8], which is a suitable method to investigate the relationship between spectacle use and myopia progression. In this study, with the data from the MYOSOTIS survey [9], PSM is applied to construct two groups of similar children with low myopia and different state of spectacle use, to explore its effects on myopia progression.

\section{Methods}

\section{Study design}

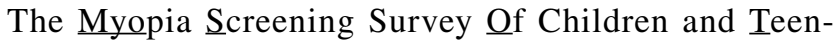

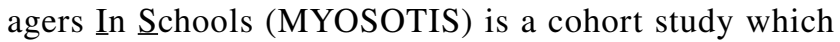
enrolled all the 46 primary and junior high schools in two districts (Shang Cheng and Bin Jiang) of Hangzhou, China. The first participants were examined in early 2019, with examinations performed at around 6 monthly intervals thereafter. We examined 44,187 students at baseline, which accounted for $95.0 \%$ of all 46,518 students in the surveyed schools. By now, four rounds of the survey have been completed. Considering that the COVID-19 pandemic lockdown was implemented between the round 2 and 3 surveys, which may bias the outcomes, only the data of the first two rounds were analyzed in this study.

The survey is supported by the National Natural Science Foundation of China (Grant No.81830027). This study was approved by the ethics committee of Eye Hospital of Wenzhou Medical University (Reference No. 2019-083-K-80) and adhered to the tenets of the Declaration of Helsinki. Informed consent was obtained from all the participants and their parents or guardians.

\section{Measurements}

Visual acuity (VA) was measured with the China's standard logarithmic eyesight chart at $5 \mathrm{~m}$ in good lighting room. In spectacle users, the corrected VA with their own spectacles was tested after the uncorrected VA. The presenting VA was defined as corrected VA in spectacle users and uncorrected VA in the others. Visual acuity in this study referred to uncorrected VA, unless otherwise noted. Refractive error was estimated by noncycloplegic autorefraction with the Tianle RM9000 autorefractometer (Ningbo Ming Sing Optical R\&D Co., Ltd, Ningbo, China). The spherical power and cylindrical power in diopter, as well as cylindrical angle, were recorded. The spherical equivalent refraction (SER) was calculated as sphere power $+1 / 2$ cylinder power. Myopic progression was defined as a more negative SER.

\section{Eligible criteria}

As shown in Fig. 1, the students who met any criteria were excluded, which contained the children who were older than 16 years or younger than 6 years at baseline or whose age was not available; the students with reported history of ortho-k or other contact lenses wearing; the participants who graduated from the enrolled schools or lost to followup at round 2; the students with missing refractive data or with extreme refractive records (astigmatism with cylinder power $>4 \mathrm{D}$, myopia with $\mathrm{SER}<-9 \mathrm{D}$, or hyperopia with SER $>5$ D) in either eye, or with anisometropia $>2$ D between two eyes, at any survey round. We also excluded those with change in SER between two rounds $>2 \mathrm{D}$ in either eye.

Of the 34,676 students remaining, 11,008 were defined as low myopia, with a mean SER of both eyes between -0.5 and $-3.0 \mathrm{D}$ and uncorrected VA $\leq 1.0 \mathrm{log}$ MAR in both eyes at round 1 . The students were categorized into two groups: group 1 (non-glasses users at round 1) and group 2 (spectacle users at both survey rounds). After 1-to-1 propensity score matching and within a 
Fig. 1 Flow chart showing the students included for analysis. D, diopter; SER, spherical equivalent refraction

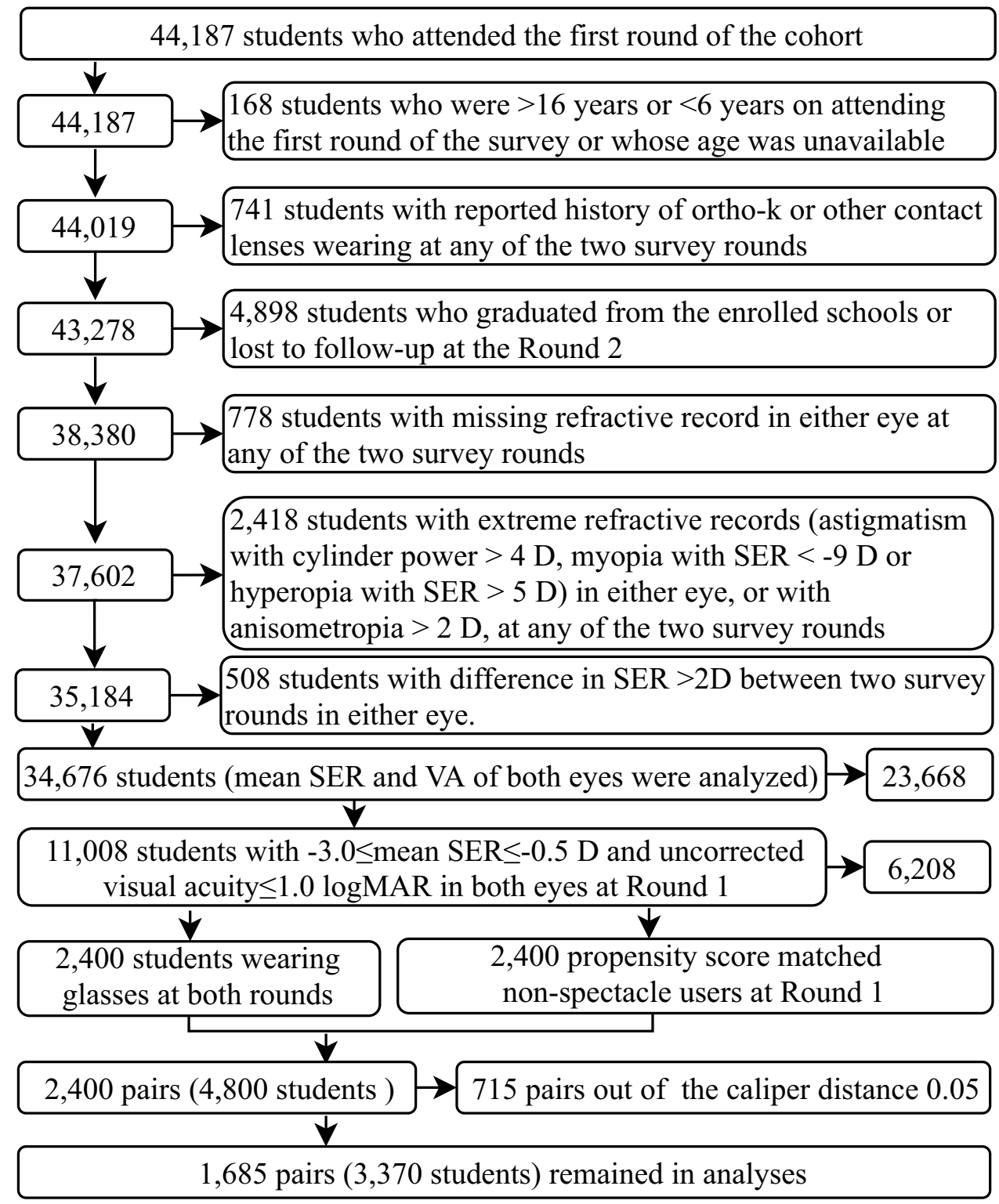

caliper distance of 0.05 , a total of 1,685 pairs $(3,370$ students) were enrolled in analyses.

\section{Statistical analysis}

Age and time interval were calculated in days and analyzed as continuous variables. The rate of SER change ( $r \Delta$ SER) was calculated as the difference in SER $(\triangle \mathrm{SER})$ between two rounds divided by the corresponding time interval in year. Mean SER and VA of both eyes were analyzed in all models unless otherwise mentioned.

PSM was performed using the STATA psmatch2 package, with 1-to-1 matching between groups 1 and 2 and within a caliper of 0.05 (which is determined by $1 / 4 \times$ standard deviation of the propensity scores). $T$-test was performed to compare means and chi-square test was used to compare sex ratio between groups. Linear regression models were applied to investigate the associations between $\mathrm{r} \triangle \mathrm{SER}$, groups, and other covariates. The 3D kernel density estimation and plotting were performed with $\mathrm{R}$ v4.0.5 (The $\mathrm{R}$ Foundation), using the $k s$ package [10]. Age, SER, and VA at round 1 were used as the 3 dimensions to visualize the differences in distribution between the two groups. Subgroup analyses were performed based on tertiles of age and SER at baseline, while sensitivity analyses were performed based on eye sides. All analyses except the 3D kernel density plotting were performed using STATA/SE-15 (StataCorp LLC, College Station, TX). 


\section{Results}

As shown in Fig. 1, of the 44,187 participants at baseline, 1,685 pairs of PSM matched 3,370 students with low myopia were enrolled for analyses, and the average follow-up time was $6.3 \pm 0.4$ months for group 1 and $6.4 \pm 0.4$ months for group 2. In Table 1, before PSM, the means of all analyzed variables at baseline were significantly different between the two groups $(P<0.001)$, and the spectacle users in group 2 were significantly older, more myopic, with worse VA and faster rate of myopia progression, while the sex ratio showed no significant difference between the two groups $(P=0.060)$. After PSM, no significant difference in age, sex ratio, VA, and SER between the two groups was found at baseline,

Table 1 The descriptions of the students with low myopia at round 1 before and after propensity score matching (PSM) ${ }^{\dagger}$

\begin{tabular}{|c|c|c|c|c|c|c|}
\hline & \multicolumn{2}{|c|}{ Students with low myopia before PSM* } & \multirow[t]{2}{*}{$P$} & \multicolumn{2}{|c|}{ PS matched students with low myopia* } & \multirow[t]{2}{*}{$P$} \\
\hline & Group $1(n=7,663)$ & Group $2(n=2,400)$ & & Group $1(n=1,685)$ & Group $2(n=1,685)$ & \\
\hline Age (years) & $10.4 \pm 2.1$ & $11.2 \pm 1.9$ & $<0.001$ & $11.0 \pm 2.0$ & $11.0 \pm 1.9$ & 0.996 \\
\hline Sex (female \%) & $47.6 \%(3650 / 7663)$ & $49.8 \%(1196 / 2400)$ & 0.060 & $47.9 \%(807 / 1685)$ & $50.1 \%(844 / 1685)$ & 0.202 \\
\hline SER (diopters) & $-1.16 \pm 0.61$ & $-2.04 \pm 0.63$ & $<0.001$ & $-1.86 \pm 0.63$ & $-1.84 \pm 0.61$ & 0.306 \\
\hline Uncorrected visual acuity (logMAR) & $0.21 \pm 0.22$ & $0.53 \pm 0.21$ & $<0.001$ & $0.45 \pm 0.18$ & $0.44 \pm 0.16$ & 0.117 \\
\hline Presenting visual acuity (logMAR) & $0.21 \pm 0.22$ & $0.09 \pm 0.14$ & $<0.001$ & $0.45 \pm 0.18$ & $0.07 \pm 0.13$ & $<0.001$ \\
\hline Propensity score & $0.15 \pm 0.19$ & $0.52 \pm 0.26$ & $<0.001$ & $0.41 \pm 0.22$ & $0.40 \pm 0.21$ & 0.096 \\
\hline Rate of SER change (diopter/year) & $-0.35 \pm 1.04$ & $-0.70 \pm 0.81$ & $<0.001$ & $-0.67 \pm 0.97$ & $-0.69 \pm 0.81$ & 0.448 \\
\hline
\end{tabular}

${ }^{\dagger}$ Continuous data in format, mean \pm standard deviation. *Group 1: non-spectacle users at round 1; group 2: spectacle users at both survey rounds. $S E R$, spherical equivalent refraction; $P S$, propensity score; $P S M$, propensity score matching. 945 students with missing information of spectacle use were not analyzed

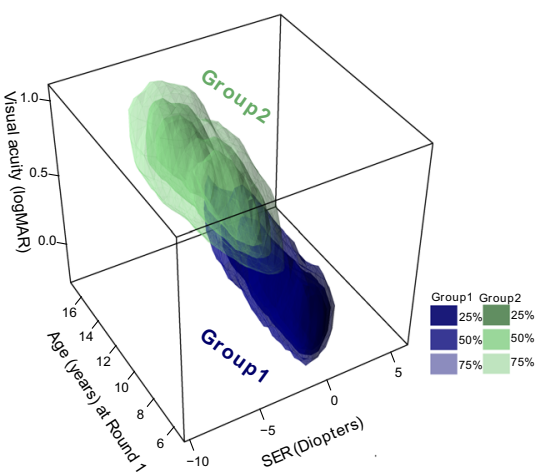

(A)

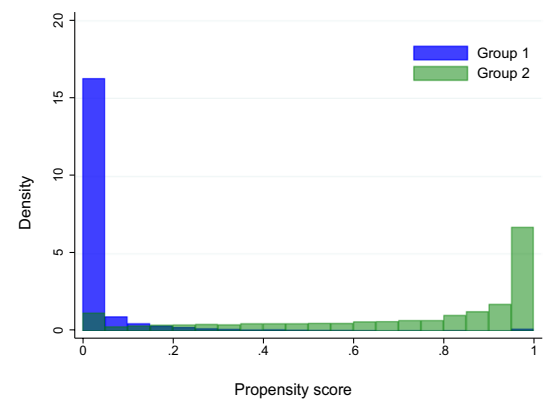

(D)

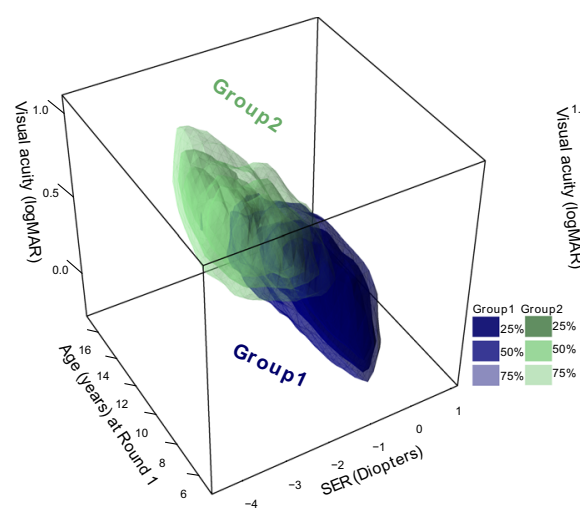

(B)

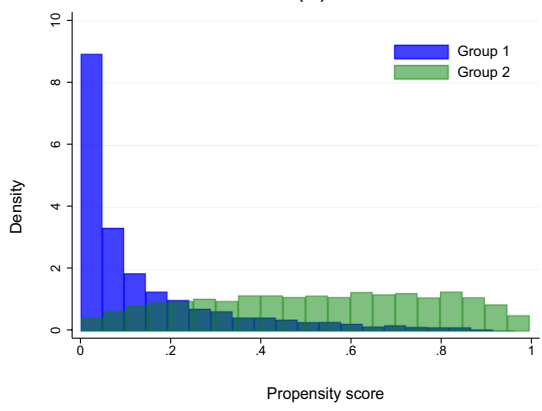

(E)

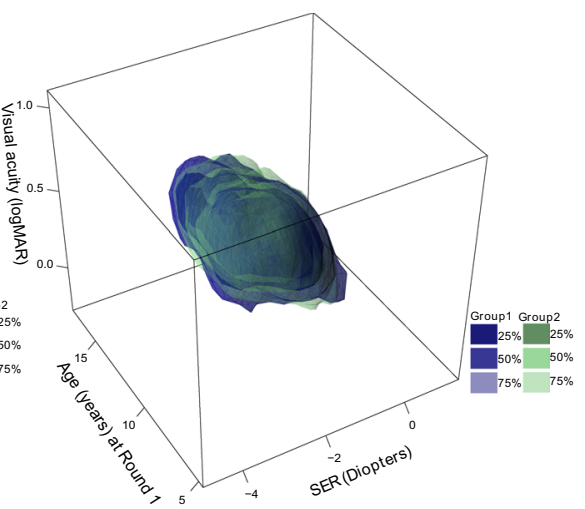

(C)

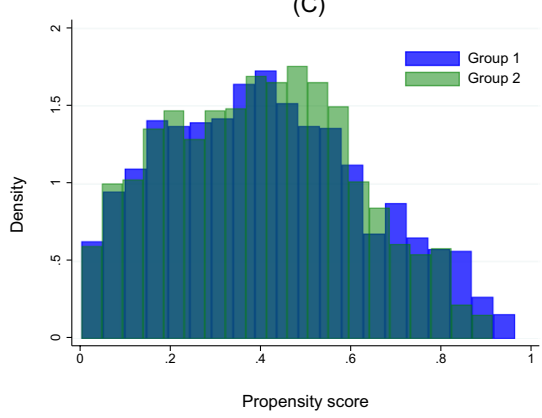

(F)
Fig. 2 The distributions of groups 1 and 2 visualized by (A-C) 3D kernel density plots with age, SER, and visual acuity at baseline as the dimensions, and (D-F) distribution plots of propensity scores. The procedure of propensity score matching (PSM) was shown as (A, D) participants who remained in analyses before applying restriction of low myopia in Fig. 1; (B, E) participants with low myopia before PSM; and (C, F) participants with low myopia after PSM (group 1: non-spectacle users at round 1; group 2: spectacle users at both survey rounds) 
while presenting VA was significantly better in spectacle users of group $2(P<0.001)$. The rate of myopia progression ( $\mathrm{r} \triangle \mathrm{SER}$ ) was similar in groups 1 and 2 after PSM (mean $\pm \mathrm{SD},-0.67 \pm 0.97$ versus $-0.69 \pm 0.81$ diopter/year, $P=0.448$ ). Figure 2 supports the outcomes in Table 1, with the 3D kernel density plots and the distribution plots of propensity scores indicating distinct difference between the two groups before applying restriction of low myopia (A, D), less distinct difference in low myopia children before PSM (B, E), and similar distributions after applying PSM (C, F).

In Table 2, the association between the rate of myopia progression and spectacle use was investigated with linear regression models (model I adjusted for age and sex at baseline; model II adjusted for age, sex, and SER at baseline; and model III further adjusted for VA at baseline). After PSM, r $\triangle$ SER showed no significant groups 1 and 2 was found in all three models, neither in right eyes nor in left eyes.

In Table S2, we subdivided group 1 (non-spectacle users at baseline), into group 1-1 (children who remained uncorrected at round 2) and group 1-2 (children who became spectacle users at round 2). Their paired students in group 2 were subdivided into group 2-1 (matches to those in group 1-1) and group 2-2 (matches to those in group 1-2). As shown in Table S2, the $\mathrm{r} \triangle \mathrm{SER}$ showed no significant difference between groups $2-1$ and $2-2$, while significant slower rate in group 1-1 and faster rate in group 1-2 were found in all three models $(P<0.001)$.

\section{Discussion}

After PSM, 1,685 pairs of students with low myopia were

Table 2 Linear regression for the rate of spherical equivalent refraction change ( $\mathrm{r} \Delta \mathrm{SER}$, diopter/year), in participants with low myopia after propensity score matching (PSM)

\begin{tabular}{|c|c|c|c|c|c|c|}
\hline \multirow[t]{2}{*}{ Variables } & \multicolumn{2}{|l|}{ Model I } & \multicolumn{2}{|l|}{ Model II } & \multicolumn{2}{|l|}{ Model III } \\
\hline & Coefficient $(\beta)(95 \% \mathrm{CI})$ & $P$ & Coefficient $(\beta)(95 \% \mathrm{CI})$ & $P$ & Coefficient $(\beta)(95 \% \mathrm{CI})$ & $P$ \\
\hline Groups $(\text { Ref. }=\text { group } 1)^{*}$ & $-0.025(-0.084,0.035)$ & 0.418 & $-0.023(-0.082,0.037)$ & 0.455 & $-0.031(-0.089,0.028)$ & 0.302 \\
\hline Age (years) & $0.080(0.063,0.096)$ & $<0.001$ & $0.074(0.058,0.091)$ & $<0.001$ & $0.068(0.052,0.084)$ & $<0.001$ \\
\hline Sex $($ Ref. $=$ female $)$ & $-0.051(-0.111,0.009)$ & 0.095 & $-0.057(-0.117,0.003)$ & 0.061 & $-0.079(-0.138,-0.020)$ & 0.009 \\
\hline SER (diopter) & - & - & $-0.097(-0.146,-0.049)$ & $<0.001$ & $-0.163(-0.213,-0.112)$ & $<0.001$ \\
\hline Visual acuity (logMAR) ${ }^{\dagger}$ & - & - & - & - & $-0.970(-1.160,-0.780)$ & $<0.001$ \\
\hline
\end{tabular}

*Group 1: non-spectacle users at round 1; group 2: spectacle users at both survey rounds

${ }^{\dagger}$ Uncorrected visual acuity

difference between the two groups in all three models (according to model III, the difference was $-0.031,95 \%$ $\mathrm{CI},-0.089 \sim 0.028, P=0.302$ ). For covariates, the myopia progression was significantly faster in younger, less myopic participants with worse VA. In model III, 1 year younger, one diopter less myopic, and $0.1 \log$ MAR worse VA were respectively associated with 0.068 (95\% CI, $0.052 \sim 0.084$ ), 0.163 (95\% CI, 0.112 0.213), and 0.097 (95\% CI, $0.078 \sim 0.116)$ diopter/year faster myopic progression $(P<0.001)$.

We then performed sensitivity analyses by stratifying the participants into nine subgroups based on tertiles of age and SER at baseline. As shown in Fig. 3, no significant difference in $\mathrm{r} \triangle \mathrm{SER}$ between groups 1 and 2 was found in eight subgroups $(P>0.05)$. In the remaining subgroup $(10<\mathrm{ag}$ $\mathrm{e} \leq 12$ years, $-2.15<\mathrm{SER} \leq-3.0 \mathrm{D})$, myopia progression seemed slower in group $2(P<0.05)$. However, the difference is not significant if multi-comparisons were taken into consideration (Bonferroni-corrected level). In Table S1, we further performed sensitivity analyses by sides of the eyes. Similarly, no significant difference in $\mathrm{r} \Delta \mathrm{SER}$ between enrolled into analyses. Table 1 and Fig. 2 show that PSM successfully eliminated the significant differences in age, SER, and VA between the two groups at baseline, which reduced the possible bias due to confounding variables. Our study indicated no significant association between singlevision spectacle use and myopia progression, and the conclusion was consistent in all sensitivity analyses.

In this study, PSM was performed to reduce intergroup variance. In Fig. 2, we innovatively introduced 3D kernel density plotting into PSM to visualize its effects, with the R $k s$ package [10]. Although Table 1 demonstrates that PSM successfully eliminated the difference in means of all variables for modeling, Fig. 2 further reveals that the co-distribution of the variables also becames similar. This technique can be useful in future PSM studies.

We found no significant difference in the rate of myopia progression between the two groups, which indicates that spectacle use will not accelerate nor retard myopia development. The conclusion is robust, since we got consistent conclusion in the subgroup analyses stratified by refractive status and age (Fig. 3), as well as in the sensitivity analyses 
Fig. 3 Comparison of rate of SER change ( $r \Delta \mathrm{SER})$ between groups 1 and 2 in subgroups stratified by age and SER at tertiles (group 1: non-spectacle users at round 1 ; group 2: spectacle users at both survey rounds. $P$-values of regression models: P1, adjusting for sex and age; P2, adjusting for sex, age, and SER; P2, adjusting for sex, age, SER, and visual acuity)

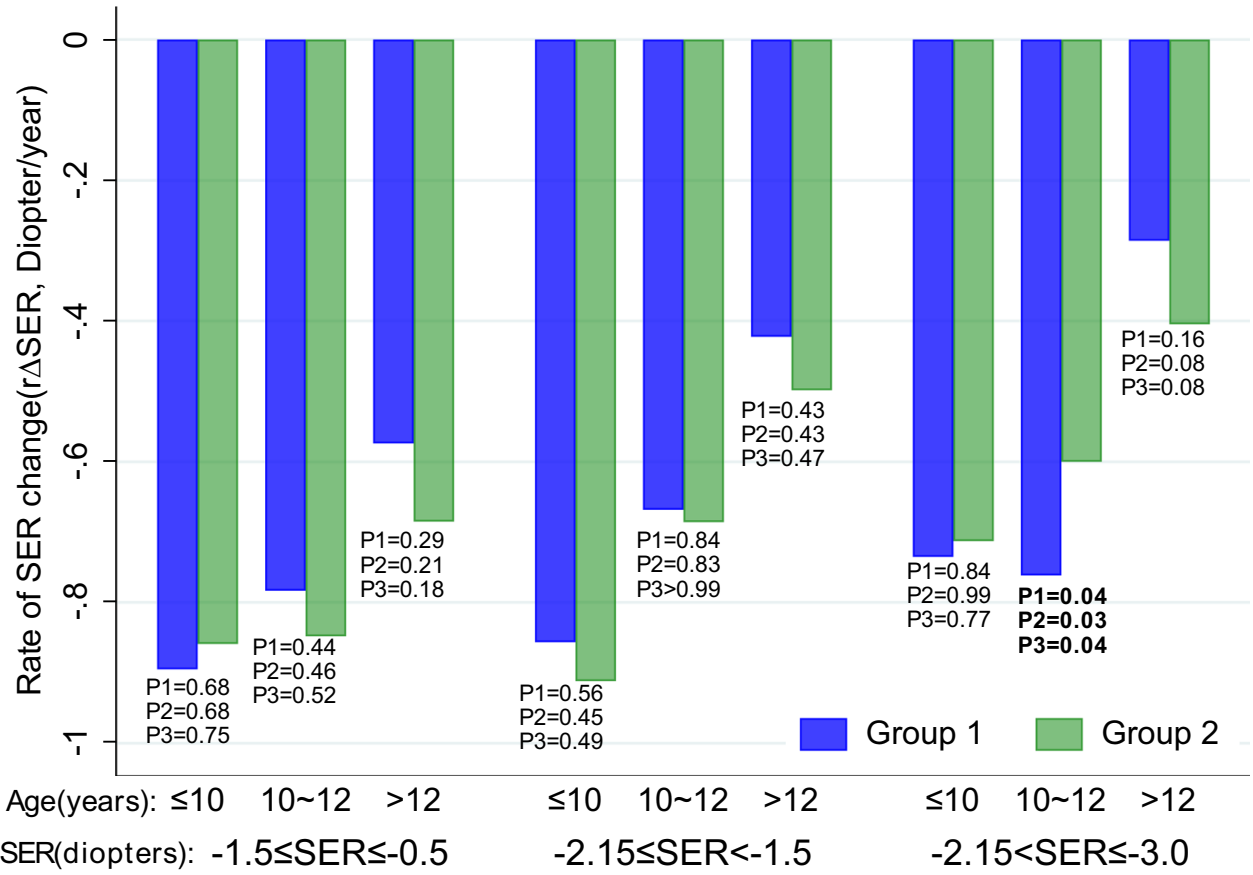

theoretical foundation to support an impact of spectacle use on myopia progression, and our study supports no association between them.

We may give some explanations on our grouping and matching methods. Firstly, we enrolled the participants into the following two groups: group 1 consisted of non-spectacle users at baseline and group 2 included spectacle users at both survey rounds. Some may doubt why not to enroll non-spectacle users at both rounds as group 1 if we hope to explore the effect of spectacle use. As shown in Table S2, for non-spectacle users at baseline, those who remained uncorrected during follow-up (group 1-1) were more likely to show slower myopia progression, while the children who became spectacle users (group 1-2) tended to present much faster myopic development. Interestingly, for their matches in group 2, no significant difference in myopia progression was found, which indicated that if only the children who remained uncorrected were enrolled as negative controls, we may artificially select those with much slower rate of myopia progression and got biased outcomes. Secondly, during PSM, we used average SER and VA of both eyes, rather than data of right, left, or a random eye. Given spectacle users tend to be more myopic with worse uncorrected VA, and the data may vary between both eyes, if only one eye is used for matching, the data of the other eye will be unbalanced, which will generate two incomparable groups.

The large sample size and well-matched participants are the major strengths of this study. However, some limitations must be considered to interpret our findings. Firstly, the refractive error was measured by noncycloplegic autorefraction, but accommodation was neglected in all analyses. 
Considering that accommodation existed at both rounds, its effects on the conclusion may be small since the study focused on the difference in SER (myopic progression). Secondly, since biometric exams were not performed in this survey, we did not have evidence on structure to support our findings. Thirdly, all participants in this study were Chinese children; it should be careful to apply the evidence in other populations.

\section{Conclusions}

In conclusion, our study indicates that single-vision spectacle use has no impact, neither acceleration nor retardation, on myopic progression in children with low myopia. Since presenting VA is significant worse in non-spectacle users, which may decrease their quality of life [6] as well as academic performance, we suggest spectacle should be prescribed in children with low myopia if their vision has been impaired.

Supplementary Information The online version contains supplementary material available at https://doi.org/10.1007/s00417-021-05423-6.

Acknowledgements We would like to express our sincere thanks to the students in this survey.

Author contribution BZ, QJ, and YEZ contributed to the design of the study. PJC, QJ, and YEZ have full access to the data in the study and take responsibility for the integrity of the data. BZ performed the data analyses. All authors contributed to data interpretation. All authors read and critically revised the manuscript. All authors approved the final manuscript.

Funding The survey is supported by the National Natural Science Foundation of China (Grant No. 81830027).

Data availability The data are not publicly available because they are containing information that may compromise research participant privacy but are available from the corresponding author on reasonable request.

Code availability Not applicable

\section{Declarations}

Ethics approval and consent to participate This study was approved by the ethics committee of Eye Hospital of Wenzhou Medical University (Reference No. 2019-083-K-80) and adhered to the tenets of the Declaration of Helsinki. Informed consent was obtained from all the participants and their parents or guardians.

Consent for publication Not applicable.

Competing interests The authors declare no competing interests.

\section{References}

1. Holden BA, Fricke TR, Wilson DA et al (2016) Global prevalence of myopia and high myopia and temporal trends from 2000 through 2050. Ophthalmology 123(5):1036-1042. https://doi.org/ 10.1016/j.ophtha.2016.01.006

2. Flaxman SR, Bourne RRA, Resnikoff S et al (2017) Global causes of blindness and distance vision impairment 1990-2020: a systematic review and meta-analysis. Lancet Glob Health 5(12):e1221e1234. https://doi.org/10.1016/s2214-109x(17)30393-5

3. García MG, Breher K, Ohlendorf A et al (2020) To correct or not correct? Actual evidence, controversy and the questions that remain open. J Clin Med 9(6):1975. https://doi.org/10.3390/jcm90 61975

4. Jackson E (1892) The full correction of myopia. Trans Am Ophthalmol Soc 6:359-373

5. Walline JJ, Lindsley KB, Vedula SS et al (2020) Interventions to slow progression of myopia in children. Cochrane Database Syst Rev 1:CD004916. https://doi.org/10.1002/14651858.CD004916. pub4

6. Kumaran SE, Balasubramaniam SM, Kumar DS et al (2015) Refractive error and vision-related quality of life in South Indian children. Optom Vis Sci 92(3):272-278. https://doi.org/10.1097/ opx.0000000000000494

7. Ma X, Zhou Z, Yi H et al (2014) Effect of providing free glasses on children's educational outcomes in China: cluster randomized controlled trial. BMJ 349:g5740. https://doi.org/10.1136/bmj. g5740

8. Haukoos JS, Lewis RJ (2015) The propensity score. JAMA 314(15):1637-1638

9. Chang P, Zhang B, Lin L et al (2021) Comparison of myopic progression before, during, and after COVID-19 lockdown. Ophthalmology. https://doi.org/10.1016/j.ophtha.2021.03.029

10. Duong T (2007) ks: kernel density estimation and kernel discriminant analysis for multivariate data in R. J Stat Softw 21(7):1-16

11. Adler D, Millodot M (2006) The possible effect of undercorrection on myopic progression in children. Clin Exp Optom 89(5):315321. https://doi.org/10.1111/j.1444-0938.2006.00055.x

12. Koomson NY, Amedo AO, Opoku-Baah C et al (2016) Relationship between reduced accommodative lag and myopia progression. Optom Vis Sci 93(7):683-691. https://doi.org/10.1097/opx. 0000000000000867

13. Li SY, Li SM, Zhou YH et al (2015) Effect of undercorrection on myopia progression in 12-year-old children. Graefes Arch Clin Exp Ophthalmol 253(8):1363-1368. https://doi.org/10.1007/ s00417-015-3053-8

14. Yan L, Huibin L, Xuemin L (2014) Accommodation-induced intraocular pressure changes in progressing myopes and emmetropes. Eye 28(11):1334-1340. https://doi.org/10.1038/eye.2014. 208

15. Ha A, Kim YK, Park YJ et al (2018) Intraocular pressure change during reading or writing on smartphone. PLoS One 13(10):e0206061. https://doi.org/10.1371/journal.pone.0206061

16. Jenssen F, Krohn J (2012) Effects of static accommodation versus repeated accommodation on intraocular pressure. J Glaucoma 21(1):45-48. https://doi.org/10.1097/IJG.0b013e31820277a9

17. Read SA, Collins MJ, Becker H et al (2010) Changes in intraocular pressure and ocular pulse amplitude with accommodation. $\mathrm{Br}$ J Ophthalmol 94(3):332-335. https://doi.org/10.1136/bjo.2009. 166355

18. Gwiazda J, Bauer J, Thorn F et al (1995) A dynamic relationship between myopia and blur-driven accommodation in school-aged children. Vision Res 35(9):1299-1304. https://doi.org/10.1016/ 0042-6989(94)00238-h 
19. Chen Y, Drobe B, Zhang C et al (2020) Accommodation is unrelated to myopia progression in Chinese myopic children. Sci Rep 10(1):12056. https://doi.org/10.1038/s41598-020-68859-6

20. Mutti DO, Mitchell GL, Hayes JR et al (2006) Accommodative lag before and after the onset of myopia. Invest Ophthalmol Vis Sci 47(3):837-846. https://doi.org/10.1167/iovs.05-0888

21. Berntsen DA, Sinnott LT, Mutti DO et al (2011) Accommodative lag and juvenile-onset myopia progression in children wearing refractive correction. Vision Res 51(9):1039-1046. https://doi. org/10.1016/j.visres.2011.02.016

Publisher's note Springer Nature remains neutral with regard to jurisdictional claims in published maps and institutional affiliations. 